\title{
Galaxy clusters as mirrors of the distant Universe
}

\section{Implications of the blurring term for the $\mathrm{kSZ}$ and ISW effects}

\author{
C. Hernández-Monteagudo ${ }^{1}$ and R. A. Sunyaev ${ }^{1,2}$ \\ 1 Max-Planck-Institut für Astrophysik, Karl-Schwarzschild-Str. 1, 85741 Garching bei München, Germany \\ e-mail: chm@mpa-garching.mpg.de \\ 2 Space Research Institute, Russian Academy of Sciences, Profsoyuznaya 84/32, 117997 Moscow, Russia \\ e-mail: sunyaev@mpa-garching.mpg.de
}

Received 19 May 2009 / Accepted 26 October 2009

ABSTRACT

\begin{abstract}
It is well known that Thomson scattering of cosmic microwave background (CMB) photons in galaxy clusters introduces new anisotropies in the $\mathrm{CMB}$ radiation field, but still little attention is payed to the fraction of $\mathrm{CMB}$ photons that are scattered off the line of sight, causing a slight blurring of the $\mathrm{CMB}$ anisotropies present at the moment of scattering. In this work we study this blurring effect and find that it can provide an independent measurement of the cluster's gas mass. Likewise, this effect has a non-negligible impact on estimations of the kSZ effect: it induces a 10\% correction in 20-40\% of the clusters/groups and a dominant over kSZ correction in $\sim 5 \%$ of the clusters in an ideal (noiseless) experiment. For rich clusters, CMB, tSZ and X-ray observations can provide estimates for the amplitude and sign of the blurring effect that can be used for correcting kSZ estimations. We explore the possibility of using this blurring term to probe the CMB anisotropy field at different epochs in our Universe. In particular, we study the required precision in the removal of the kSZ which enables us to detect the blurring term $-\tau_{\mathrm{T}} \delta T / T_{0}$ in galaxy cluster populations placed at different redshift shells. By mapping this term in those shells, we provide a tomographic probe for the growth of the Integrated Sachs-Wolfe effect (ISW) during the late evolutionary stages of the Universe. We find that the required precision on the removal of the cluster's peculiar velocity is of the order of $100-200 \mathrm{~km} \mathrm{~s}^{-1}$ in the redshift range $0.2-0.8$, after assuming that all clusters more massive than $10^{14} h^{-1} M_{\odot}$ are observable. These errors are comparable to the total expected linear line of sight velocity dispersion for clusters in WMAPV cosmogony and correspond to a residual level of roughly $900-1800 \tau_{\mathrm{T}} \mu \mathrm{K}$ per cluster, including all types of contaminants and systematics. Were this precision requirement achieved, then independent constraints on the intrinsic cosmological dipole would be simultaneously provided.
\end{abstract}

Key words. cosmic microwave background - large-scale structure of Universe

\section{Introduction}

The study of the scattering of cosmic microwave background (CMB) photons in moving clouds of thermal electrons (like galaxy clusters) has been a subject of active research since the first works of Sunyaev \& Zeldovich (1970, 1972, 1980, 1981); Zeldovich \& Syunyaev (1980). Indeed, this mechanism is one of the key two sources for intensity and polarization anisotropies in the CMB (the other one being associated to the presence of gravitational fields, the so-called Sachs-Wolfe effect, Sachs $\&$ Wolfe 1967). The Thomson scattering changes the angular distribution of the $\mathrm{CMB}$ photons, partially erasing the original anisotropy pattern (due to the off scattering of photons propagating initially along the line of sight) and introducing new anisotropies if the electrons move with respect to the CMB rest frame. Due to its anisotropic nature, Thomson scattering also introduces linear polarization if the $\mathrm{CMB}$ shows an intensity quadrupole at the scattering place. The use of the polarization induced by Thomson scattering in galaxy clusters has been proposed as a probe for remote CMB quadrupoles (Sunyaev \& Zeldovich 1980; Kamionkowski \& Loeb 1997; Sazonov \& Sunyaev 1999; Challinor et al. 2000), with implications for the integrated Sachs-Wolfe effect (ISW, Cooray \& Baumann 2003) and for the characterization of the large scale density distribution in the observable universe (e.g., Seto \& Sasaki 2000; Abramo \& Xavier 2007).

However, this work will be devoted to the study of a blurring term, i.e., the term responsible for the smearing of the intensity anisotropies of the radiation field generated early enough, either at recombination or during reionization, or before the phase of accelerated expansion of the universe. For an axisymmetric radiation field described by $T(\mu) / T_{0}=\sum_{l} a_{l} P_{l}(\mu)$ (with $\mu \equiv \cos \theta$ measuring deviations from the symmetry axis), the blurring term will affect all multipoles, whereas the anisotropic scattering will slightly modify the quadrupole $\left(a_{2}\right)$ and generate a secondary polarization (e.g. Sunyaev \& Zeldovich 1980; Zeldovich \& Syunyaev 1980; Sunyaev \& Zeldovich 1981):

$$
\frac{\Delta T}{T_{0}}=\tau_{\mathrm{T}}\left(-\sum_{l=1}^{\infty} a_{l} P_{l}(\mu)+\frac{1}{10} a_{2} P_{2}(\mu)\right),
$$

where $a_{l=1}$ refers to the intrinsic cosmological dipole at the scattering place. This dipole must not be confused with the dipole observed by COBE and WMAP, which is mainly caused by the observer's local peculiar motion. The blurring term is described by the right hand side of the equation above (note that the 
quadrupole is effectively suppressed by a factor of only $0.9 \tau_{\mathrm{T}}$ ). However, if the scatterers show a peculiar motion with respect to the radiation field, an additional Doppler term must be added to the previous expression:

$$
\frac{\Delta T}{T_{0}}=\tau_{\mathrm{T}}\left(-\sum_{l=1}^{\infty} a_{l} P_{l}(\mu)+\frac{1}{10} a_{2} P_{2}(\mu)-\frac{\boldsymbol{v} \cdot \hat{\boldsymbol{n}}}{c}\right),
$$

with $\hat{\boldsymbol{n}}$ the unit vector along the line of sight and $\boldsymbol{v}$ the scatterer's proper peculiar velocity. Since $\boldsymbol{v} \cdot \hat{\boldsymbol{n}} / c$ is typically larger than $\Delta T / T_{0}\left(\boldsymbol{v} \cdot \hat{\boldsymbol{n}} / c \simeq 7 \times 10^{-4}\right.$ in the linear approximation for WMAPV cosmology, $\Delta T / T_{0} \sim 2 \times 10^{-5}$ as measured by COBE and WMAP), the blurring term should typically correct by a few percent the peculiar motion effect. This will be hereafter denoted as the kinetic effect (Sunyaev \& Zeldovich 1980). However, the ratio between the $\mathrm{kSZ}$ and the blurring effect does depend on the cluster's peculiar motion, angular size and position in the sky, as we shall show later. At any rate, we show that the blurring term (hereafter denoted as bSZ effect) has potentially a relevant cosmological significance: apart from the impact it has on accurate measurements of the kSZ effect, it can also provide an alternative measurement of the gas mass in clusters, independently of the total thermal energy of the gas obtained from observations of the thermal S-Z effect, (Sunyaev \& Zeldovich 1972, hereafter denoted as tSZ effect). Likewise, if the Thomson scattering optical depth associated to the cluster is known, by using $\mathrm{CMB}$ observations (like those of $\mathrm{WMAP}^{1}$ or Planck ${ }^{2}$ ) this effect (and its sign) can be accurately predicted as an attenuation of the CMB anisotropy in the direction of the cluster. This effect will be significantly more important along those directions towards the dimmest and brightest spots of the measured CMB sky and will be uncorrelated to the amplitude of the $\mathrm{kSZ}$ in clusters, so in some cases these two effects will add up while in others they will partially cancel each other.

If detected on a set of cluster samples placed at different redshifts, the bSZ term can be used as a probe in situ of the CMB anisotropy field at those epochs. By looking at the variations of this term in different redshift shells, one should be able to track the growth of new anisotropies arising at later times. In particular, it should enable us to perform tomography of the ISW effect, generated by the decay of the linear gravitational potentials at late epochs.

This acquires particular relevance in the context of current SZ cluster surveys like SPT (Staniszewski et al. 2009), ACT (Hincks et al. 2009) or Planck, and future surveys like the X-ray mission SPECTRUM-X/eROSITA ${ }^{3}$, whose prospect is to locate $\sim 150000$ galaxy clusters in the sky (among them all clusters above $2 \times 10^{14} M_{\odot}$ in the observable Universe). Some of these clusters will sit on top of high amplitude CMB intensity excursions where this effect can be measured more easily. Furthermore, we also show that this phenomenon should also provide stringent limits on the amplitude of the intrinsic cosmological dipole.

This paper is organized as follows: in Sect. 2 we describe the blurring term within the context of Thomson scattering. In Sect. 3 we study its implications in the measurement of $\mathrm{kSZ}$ and remote quadrupoles at the position of galaxy clusters.

\footnotetext{
1 WMAP's URL site:

http: lambda.gsfs . nasa.gov/product/map/current/

2 Planck's URL site:

http://www.esa.int/esaMI/Planck/index.html

3 Spectrum-X/eROSITA's URL site:

http://www . mpe.mpg.de/projects.html\#erosita
}

In Sect. 4 we introduce the possibility of using the blurring term for tracking the growth of the ISW: we analyse the requirements in the cluster sample and in the peculiar velocity recovery. We observe the possibility of setting constraints on the cosmological dipole by using this effect in Sect. 5. Finally, in Sect. 6 we discuss our results and conclude.

\section{The scattering of CMB photons in electron clouds}

Thomson scattering modifies the angular pattern of the CMB intensity and polarization anisotropies. The source for new intensity anisotropies is associated with the peculiar velocity of the gas cloud with respect to the CMB frame (Sunyaev \& Zeldovich 1980), whereas in the case of polarization anisotropies it is associated with the CMB quadrupole at the scattering place. If the electron gas is at a high temperature, then Compton scattering transfers energy from the electron plasma to the CMB photon field, distorting the $\mathrm{CMB}$ black body spectrum and introducing frequency dependent temperature fluctuations (tSZ effect). The tSZ effect (and its relativistic corrections) has a definite spectral dependence (Sunyaev \& Zeldovich 1972, 1981; Rephaeli 1995; Itoh \& Nozawa 2004), so hereafter we shall assume that it can be accurately subtracted.

Our interest in this paper will be focused on the intensity blurring term $\left(-\tau_{\mathrm{T}} \delta T(\hat{\boldsymbol{n}}) / T_{0}\right)$, which accounts for the fraction of photons that, initially propagating along the line of sight, were scattered off it and never reach the observer. This term hence describes the erasing of the CMB anisotropies at the scattering position along the line of sight towards the electron cloud, since the Thomson scattering tends to isotropize the CMB angular fluctuations in that direction. This adds up to the kSZ effect, with no distinction on the photon's frequency and hence preserving the CMB black body spectrum. The source of the polarization, instead, is the cluster's local CMB intensity quadrupole, which is sensitive to the CMB at all directions in that position. In the following considerations we regard the galaxy cluster and group population as clouds of free electrons.

\subsection{Estimating the blurring effect}

Both bSZ and kSZ have exactly the same spectral dependence, and this complicates their separation. However, current and future multifrequency CMB observations should provide estimates of the tSZ at each cluster's position. Indeed, tSZ measurements are currently being provided by experiments like SPT, ACT, BIMA, CBI, SZA, AMI, or AMIBA in more than a hundred galaxy clusters. By combining these measurements with $\mathrm{X}$-ray derived estimates of the gas temperature ( $T_{\mathrm{e}}$, provided by, e.g., CHANDRA or XMM) one can find the cluster's optical depth $\tau_{\mathrm{T}}$,

$$
\tau_{\mathrm{T}}=\frac{\delta T /\left.T_{0}\right|_{\mathrm{tSZ}}}{g_{\mathrm{tSZ}}(x)} \frac{m_{\mathrm{e}} c^{2}}{k_{\mathrm{B}} T_{\mathrm{e}}}
$$

where the function $g_{\mathrm{tSZ}}(x)=x \operatorname{coth}(x / 2)-4$ (with $x \equiv$ $\left.h v / k_{\mathrm{B}} T_{0}\right)$ provides the frequency dependence of the tSZ-induced brightness temperature change (Zeldovich \& Sunyaev 1969). At the same time, CMB observations (like those from WMAP and Planck) can provide measurements of the background $\mathrm{CMB}$ temperature field. Once the background CMB temperature anisotropies and the Thomson optical depth of the cluster 
C. Hernández-Monteagudo and R. A. Sunyaev: Galaxy clusters as mirrors of distant Universe

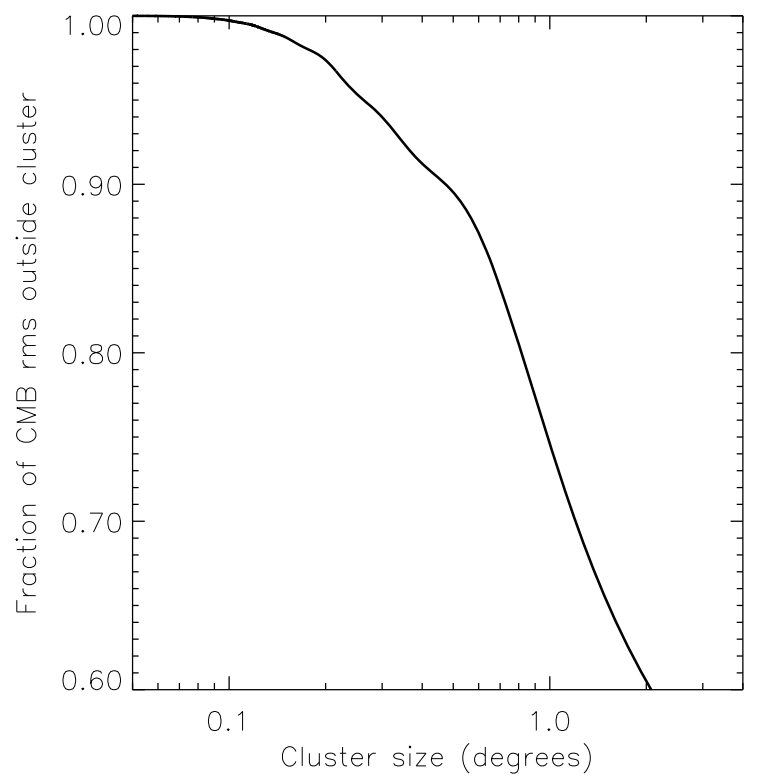

Fig. 1. Fraction of the total $\mathrm{CMB}$ temperature rms that corresponds to scales larger than the cluster size. The change of slope at $\theta \simeq 0.6^{\circ}$ is due to the acoustic peak structure in the $\mathrm{CMB}$ angular power spectrum. Galaxy clusters at cosmological distances have angular sizes smaller than $0.1^{\circ}$, and therefore the bSZ can be predicted by a CMB map of a resolution worse than the cluster size.

are known, the amplitude and the sign of the bSZ effect can be predicted:

$\left.\frac{\delta T}{T_{0}}\right|_{b}=-\left.\tau_{\mathrm{T}} \frac{\delta T}{T_{0}}\right|_{\text {intr }}$,

with $\delta T /\left.T_{0}\right|_{\text {intr }}$ the intrinsic $\mathrm{CMB}$ anisotropies. Its amplitude will be larger for those regions hosting larger excursions of the $\mathrm{CMB}$ intensity field and should dominate the kSZ for those clusters having little radial relative motion with respect to the CMB. In reality, these fluctuations will be smeared by the finite area of the cluster, but as we show in Fig. 1 this has little effect for clusters placed at cosmological distances and subtending angles below $0.1^{\circ}$. In the full sky, for the WMAPV universe, the CMB intensity rms amplitude is $\sigma_{\mathrm{CMB}} \simeq 114 \mu \mathrm{K}$. This means that, for a survey of $\sim 150000$ clusters (as the one to be conducted by SPECTRUM-X/eROSITA), approximately ten clusters will show decrements of amplitude larger than $470 \tau_{\mathrm{T}} \mu \mathrm{K}$ $(4 \sigma)$, and $\sim 400$ clusters will be above the level of $350 \tau_{\mathrm{T}} \mu \mathrm{K}$, $(3 \sigma)$. These estimates show that for $\tau_{\mathrm{T}}=10^{-2}$ the bSZ should excess the one micro Kelvin level, which is still several times below the sensitivity of the majority of existing CMB instruments. Finally, it is worth to remark that by predicting and removing the bSZ estimates one would produce more accurate measurements of the kSZ.

When averaging over the cluster's area, it is relevant to know the amplitude of the rms CMB fluctuations which is being blurred by the cluster. This can be computed by simply adding up the contribution of different angular scales or multipoles to the $\mathrm{CMB}$ variance up to the cluster scale,

$\sigma_{\mathrm{CMB}}^{2}\left[l_{\mathrm{cl}}\right]=\sum_{l=1}^{l=l_{\mathrm{cl}}} \frac{2 l+1}{4 \pi} C_{l}$,

where $l_{\mathrm{cl}}=\pi / \theta_{\mathrm{cl}}$ and $\theta_{\mathrm{cl}}$ is the cluster angular size, and we are considering the intrinsic cosmological dipole. The fraction of the
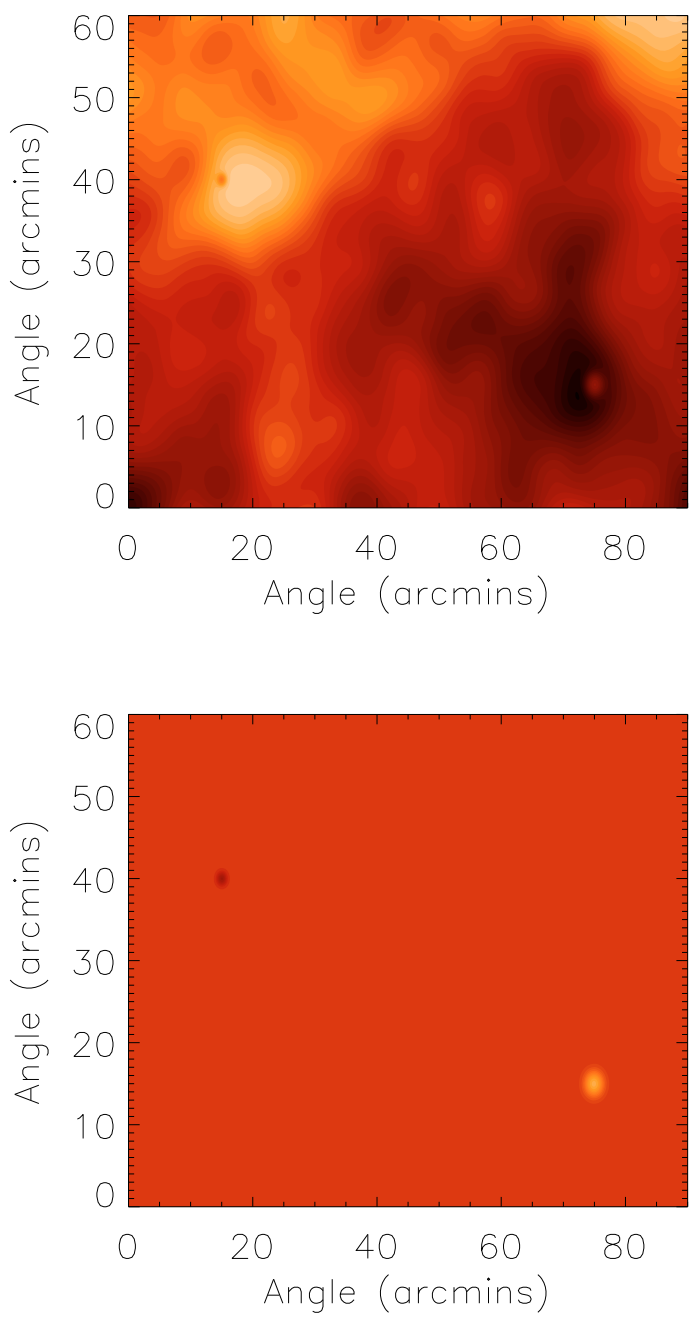

Fig. 2. Top panel: total CMB map with two clusters for which the tSZ and the kSZ contributions have been removed. A high value of $\tau_{\mathrm{T}}$ $\left(\tau_{\mathrm{T}}=0.8\right)$ was chosen for illustration purposes. Bottom panel: isolated contribution of the bSZ from the clusters: the left cluster is sitting on an intrinsic CMB bright spot and hence gives rise to a negative bSZ, whereas the right cluster is aligned with a CMB trough and its bSZ introduces a relative enhancement of the CMB brightness.

total CMB rms being blurred by the cluster can be easily computed as $\sigma_{\mathrm{CMB}}\left[l_{\mathrm{cl}}\right] / \sigma_{\mathrm{CMB}}[\infty]$. Figure 1 shows the fraction of the total $\mathrm{CMB}$ rms which is being blurred by a cluster of a given size. The change of slope of that curve at $\sim 36$ arcmins $\left(0.6^{\circ}\right)$ is due to the acoustic peak structure. Figure 2 displays a simulated case where two clusters blur original CMB anisotropies according to the standard $\Lambda \mathrm{CDM}$ scenario. Even in a patch of relatively small dimensions $\left(1.7^{\circ} \times 1.7^{\circ}\right)$, one can easily obtain $\mathrm{CMB}$ excursions above the level of $100 \mu \mathrm{K}$.

\subsection{Sensitivity to the total electron content of the cluster}

The bSZ term can be used to measure the number of electrons present in a galaxy cluster. It depends linearly on the Thomson optical depth $\tau_{\mathrm{T}}$ and on the CMB temperature fluctuations at the place of scattering. The latter should be accurately mapped by ongoing CMB experiments like WMAP, Planck, or, at higher angular resolution, by ACT or SPT. The measurement of the bSZ at different projected distances to the cluster's center would therefore provide a useful handle on the electron content and, after 
assuming a baryon mass fraction (e.g. Ettori et al. 2009; Giodini et al. 2009; Vikhlinin et al. 2006), on the entire mass profile of the cluster. This can easily been seen by writing the baryonic mass profile of the cluster in terms of its Thomson optical depth:

$M_{b}\left(<r=\theta \times d_{\text {Ang }}\right) \simeq \frac{m_{\mathrm{p}} d_{\text {Ang }}^{2}}{\sigma_{\mathrm{T}} f_{\mathrm{e}}} \int_{n<\theta} \mathrm{d} \hat{\boldsymbol{n}} \tau_{\mathrm{T}}(\hat{\boldsymbol{n}})$,

where $d_{\text {Ang }}$ is the cluster's angular diameter distance, $m_{\mathrm{p}}$ is the proton mass, $f_{\mathrm{e}}$ is the (Helium abundance dependent) average number of electrons per hydrogen mass unit ( $f_{\mathrm{e}} \simeq 1.3$ for Helium abundance $Y \simeq 0.24)$, and $M(<r)$ is the gas mass contained within a radius $r$. This exercise is simplified if the characteristic size of the CMB temperature fluctuations are bigger than the characteristic angular size of the clusters, as shown in Fig. 2.

For the case of unresolved clusters, the bSZ would provide a measurement of the total baryon content of those objects directly. This constitutes a useful independent test for mass estimates in clusters of galaxies, which are of an utmost importance in cosmological studies of tSZ surveys.

\section{Impact on $\mathrm{kSZ}$ estimations}

Let us consider here the case where the peculiar velocity of the gas is equal to that of dark matter. Let us keep in mind, however, that bulk velocities of gas inside massive galaxy clusters may significantly exceed the peculiar speed of the entire cluster (Sunyaev et al. 2003; Inogamov \& Sunyaev 2003). As mentioned above, the kSZ effect in clusters has the same spectral dependence of the intrinsic CMB anisotropies, and therefore extracting it requires the use of spatial frequency information. Its amplitude is directly proportional to the projection of the cluster's peculiar velocity along the line of sight. In linear theory and after assuming that the initial rotational component of the peculiar velocity field is negligible (since it scales with the inverse of the cosmological expansion scale factor), it is possible to relate the Fourier modes of the peculiar velocity with those of the matter density field $\left(\delta_{k}\right)$ :

$\boldsymbol{v}_{\boldsymbol{k}}=-i H(z) \frac{\mathrm{d} \mathcal{D}_{\delta}(z)}{\mathrm{d} z} \frac{\delta_{\boldsymbol{k}}}{k^{2}} \boldsymbol{k}$,

where $H(z)$ is the Hubble function and $\mathcal{D}_{\delta}(z)$ is the linear growth factor for the density perturbations. After Fourier transforming into real space, it is easy to prove that the three spatial components of the peculiar velocity are uncorrelated, i.e., the tensor $\left\langle v_{i}(\boldsymbol{x}) v_{j}(\boldsymbol{x})\right\rangle$ is diagonal $\left(\propto \delta_{i j}\right)$ for $i=1,2,3^{4}$. A direct consequence of this is that studying the line of sight projected component of the real space peculiar velocity is totally equivalent to studying any of its three components. From Eq. (7) it is easy to infer that in linear theory velocities will be Gaussian distributed and each component will have a dispersion given by

$\sigma_{v}^{2}(M)=\frac{1}{3} \int \frac{\mathrm{d} \boldsymbol{k}}{(2 \pi)^{3}} H^{2}(z)\left|\frac{\mathrm{d} \mathcal{D}_{\delta}}{\mathrm{d} z}\right|^{2} \frac{P_{\mathrm{m}}(k)}{k^{2}}|W(k R[M])|^{2}$,

where $W(k R[M])$ is the Fourier window function of a top hat filter of size given by the linear scale corresponding to the cluster mass $M, R=\left[3 \rho_{\mathrm{m}} /(4 \pi)\right]^{1 / 3}$, and $\rho_{\mathrm{m}}$ the matter comoving density. The linear matter power spectrum is given by $P_{\mathrm{m}}(k)$. In Fig. 3a we display the linear rms amplitude of the radial component of the peculiar velocity versus redshift in the standard

4 This is not the case in Fourier space, where different spatial components of $\boldsymbol{v}_{\boldsymbol{k}}$ are correlated.
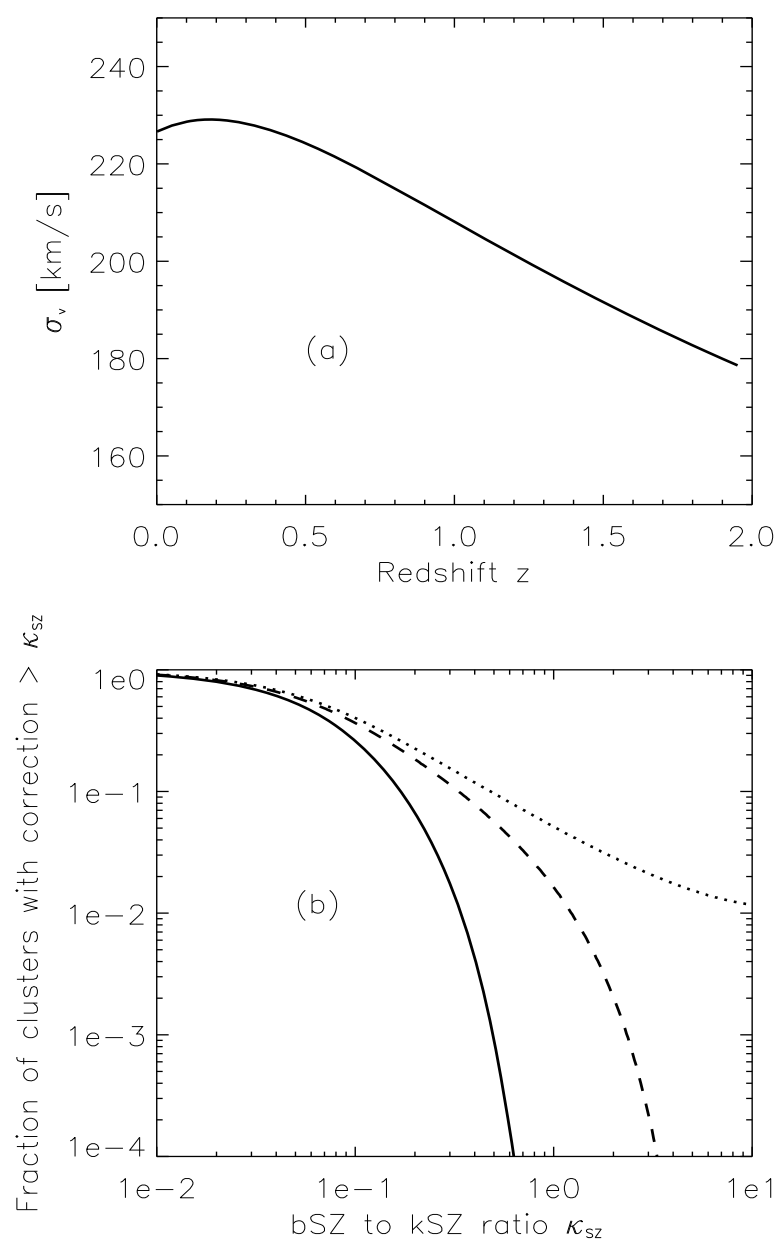

Fig. 3. a) Amplitude of the linear rms radial peculiar velocity in a WMAPV cosmology for a $2 \times 10^{14} h^{-1} M_{\odot}$ cluster of galaxies. b) Fraction of clusters in the sky above a given fractional level of correction on the kSZ induced by the bSZ term. Solid, dashed and dotted lines correspond to a level of residual (instrumental noise related) radial velocity of 60,10 and $0 \mathrm{~km} \mathrm{~s}^{-1}$.

$\Lambda \mathrm{CDM}$ scenario. In an Einstein-de Sitter (EdS) universe, its redshift scaling is $\propto 1 / \sqrt{1+z}$, but in a $\Lambda$ CDM universe its decay is even more slowly (it drops about $20 \%$ in the redshift range $z \in[0,2])$. Galaxy clusters and groups where the kSZ can be measured are biased, peculiar regions in the Universe. Different studies have attempted to quantify whether the peculiar velocities of these objects are actually biased with respect to linear theory predictions. Bardeen et al. (1986) found that their velocity should be better approximated by that of the peaks in a Gaussian distribution. Sheth \& Diaferio (2001) conducted a thorough study of peculiar velocities in simulations, in which they showed that halo peculiar velocities depend also upon the environment density, but even in the extreme cases the change in peculiar velocity would be smaller than $\sim 70 \%$ of the average value. Peel (2006) found an average bias of $\sim 30 \%$ of the halo peculiar velocity when compared to linear theory predictions.

We have adopted an average bias of $30 \%$ in the halo peculiar velocities, and estimated the average correction to the $\mathrm{kSZ}$ which the bSZ term causes. We have assumed accordingly that both the CMB temperature anisotropies and the kSZ fluctuations are Gaussian distributed and computed the fraction of clusters whose $\mathrm{kSZ}$ undergoes a correction above a certain level 
$\kappa_{\mathrm{kSZ}} \equiv\left|\delta_{\mathrm{blurr}} / \delta_{\mathrm{kSZ}}\right|=\left|\delta_{\mathrm{CMB}} /\left[(\boldsymbol{v} \cdot \hat{\boldsymbol{n}}) / c+v_{\mathrm{res}} / c\right]\right|$ due to the bSZ effect:

$$
\begin{aligned}
\mathcal{P}\left(>\kappa_{\mathrm{kSZ}}\right)= & \iint_{\left|\delta_{\mathrm{blurr}}^{\prime} / \delta_{\mathrm{kSZ}}^{\prime}\right|>\kappa_{\mathrm{kSZ}}} d\left(\frac{\boldsymbol{v} \cdot \hat{\boldsymbol{n}}}{c}\right)^{\prime} \mathrm{d} \delta_{\mathrm{CMB}}^{\prime} \\
& \times p\left(\delta_{\mathrm{CMB}}^{\prime}\right) p\left[\left(\frac{\boldsymbol{v} \cdot \hat{\boldsymbol{n}}}{c}\right)^{\prime}\right] .
\end{aligned}
$$

In both cases, the function $p(X)$ denoted the Gaussian probability distribution function on the variable $X$. The term $v_{\text {res }} / c$ is included as a residual radial velocity term which avoids divergences: the solid line in Fig. $3 \mathrm{~b}$ corresponds to $v_{\text {res }}=60 \mathrm{~km} \mathrm{~s}^{-1}$, the dashed line to $v_{\text {res }}=10 \mathrm{~km} \mathrm{~s}^{-1}$ and the dotted line to a negligible level of $v_{\text {res }}, v_{\text {res }} \simeq 0 \mathrm{~km} \mathrm{~s}^{-1}$. These residual radial velocities obey the unavoidable level of noise present at the cluster's position when observed by experiments. The level of $60 \mathrm{~km} \mathrm{~s}^{-1}$ corresponds roughly to a noise level of $1 \mu \mathrm{K}$. Figure 3 shows that, for a residual velocity of $10 \mathrm{~km} \mathrm{~s}^{-1}$, more than $30 \%(1 \%)$ of the clusters undergo a correction on the $\mathrm{kSZ}$ which is larger than $10 \%(100 \%)$. Obviously, for smaller radial velocities (and smaller values of $v_{\text {res }}$ ), the correction induced is larger. Approximately, the bSZ amplitude can be expressed as $|\delta T|_{\text {blurr }} \sim\left|\delta T_{\mathrm{CMB}} / \sigma_{\mathrm{CMB}}\right|\left(\tau_{\mathrm{T}} / 10^{-2}\right) \mu \mathrm{K}$. Yoshida et al. (2001) noted that actual probability distribution of the $\mathrm{kSZ}$ temperature fluctuations is the result of the product of two practically independent distributions: the first one corresponds to the cluster optical depth $\tau_{\mathrm{T}}$, the second one to the line of sight projected peculiar velocity. This product results in an a priori nonGaussian distribution for the kSZ intensity fluctuation. However, in our study the relevant quantity is the relative correction to the $\mathrm{kSZ}$ amplitude due to the bSZ effect. This is done on a clusterby-cluster basis, for which the value of $\tau_{\mathrm{T}}$ is, at zero order, irrelevant. In this context, the use of Gaussian distributions in Eq. (9) should be justified.

\section{Implications for the ISW effect}

By measuring the intensity blurring term through the lines of sight towards clusters placed at a given redshift shell an estimate of the CMB temperature field at that redshift is obtained. In Fig. 4a we show the CMB TT (intensity) power spectrum as measured by observers placed at different redshifts; the thick solid line corresponds to $z=0$, the dotted line to $z=0.1$, the dashed line to $z=1$ and the dot-dashed line to $z=2$. Since those observers are closer to the surface of last scattering, the whole acoustic pattern shifts to larger angular scales (smaller multipoles). Furthermore, at redshifts larger than $z \sim 1$ the contribution of the ISW is very small, and this is also visible in the low $l$ range. After the scattering, the angular pattern of the CMB anisotropies would stream unhindered towards the observer, shifting the whole picture "back" to its standard position, (see Fig. 4b). In Fig. 5 we display the free streaming of the CMB quadrupole multipole $a_{2,0}(\hat{\boldsymbol{n}})$ as seen by an observer placed at different redshifts into different multipoles $a_{l, 0}$-s. The case of redshift $z=0.1$ is displayed by solid circles joined by a solid black line, $z=1$ by red triangles joined by a dashed line, and $z=2$ by green squares joined by a dot-dashed line. The further away the remote observer is, the more power is aliased into high $l$ multipoles. This streaming of the CMB angular anisotropies permits us a comparison of the ISW pattern at different cosmological epochs on the angular/multipole scale. If the bSZ term is observed through the line of sights corresponding to a population of galaxy clusters and groups situated at a high redshift, it would provide a picture of the CMB pattern before the ISW arises. This

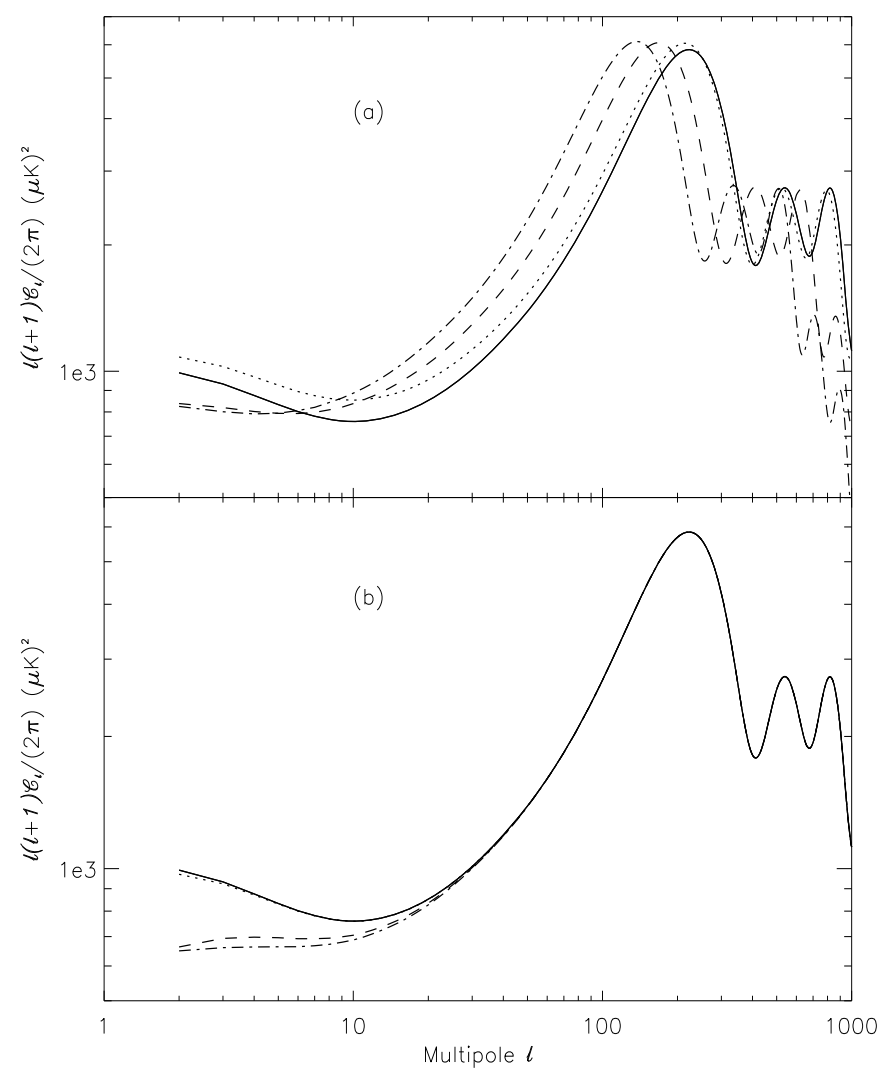

Fig. 4. a) CMB TT angular power spectrum as seen by observers placed at redshifts $z=0,0.1,1$ and 2 (solid, dotted, dashed and dot-dashed lines, respectively). b) Same power spectra as in a) after being free streamed to the present moment.

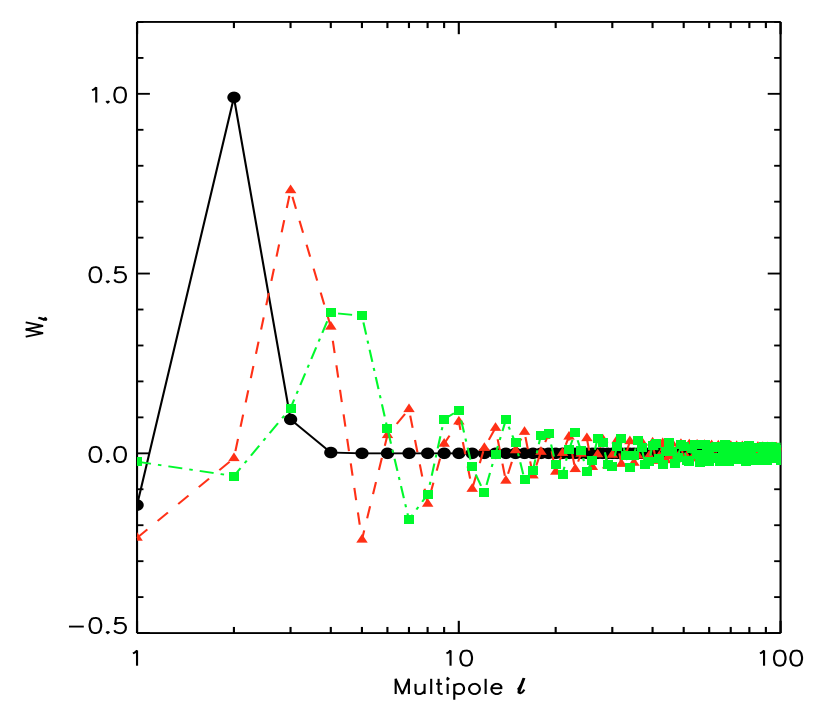

Fig. 5. Projection of the CMB quadrupole multipole $a_{2,0}$ as seen by observers placed at different redshifts into different $a_{l, 0}$ multipoles observed at present. Filled circles connected with a black solid line correspond to an observer placed at $z=0.1$, red triangles by a red dashed line to an observer at $z=1$ and green squares linked by a dot-dashed line to $z=2$.

means that, by observing the bSZ term in galaxy clusters placed at different redshift shells it should be possible, a priori, to track the growth of the ISW effect with decreasing redshift. 
In the future, several surveys in the optical and in the X-ray range (Pan-STARRS ${ }^{5}, \mathrm{DES}^{6}$, PAU-BAO ${ }^{7}$, Benitez et al. 2008, Spectrum-X/eROSITA) will probe the cosmological density field up to redshifts $z \sim 0.9-1.1$ with unprecedented sensitivity. Gravity relates the matter density distribution with the peculiar motion it causes, so a good estimation for the kSZ in clusters and groups should be obtainable from the density surveys themselves (this is indeed the goal for cosmological reconstruction algorithms like ARGO, Kitaura \& Enßlin 2008). The typical correlation length of the peculiar velocity field is $\sim 40 h^{-1}$ Mpc (comoving), which at $z \sim 1$ subtends around a couple of degrees. This means that in the large angular scales where the ISW is present we should expect to have a fairly high number of uncorrelated estimates of the cluster peculiar velocity. Let us assume that we have, for a given redshift shell, a sample of $N_{\mathrm{cl}}$ clusters in the sky. By looking only at the angular position of these clusters, we want to find out to which range of multipoles we are sensitive. It is clear that, in order to sample a given multipole $l$, our sphere tracers must lie at distances smaller than $\theta \sim \pi / l$. For a set of uniformly distributed clusters on the sphere, we can assign an area of $4 \pi / N_{\mathrm{cl}}$ to each cluster and hence an average inter-cluster separation of $\theta_{\mathrm{cc}} \simeq 2 \theta_{\mathrm{c}}=4 / \sqrt{N_{\mathrm{cl}}}$ (with $\theta_{\mathrm{c}}=2 / \sqrt{N_{\mathrm{cl}}}$ the radius assigned to each cluster area). Therefore, the maximum multipole to which our cluster sample is sensitive is given by

$l_{M} \simeq \frac{\pi}{4} \sqrt{N_{\mathrm{cl}}}$.

Let us now assume that, as justified above, the noise is uncorrelated from pixel to pixel (since the typical separation between pixels corresponds to an actual distance which is larger than the typical correlation length of the kSZ). In this case, we model the signal in our $i$ th-pixel as

$s\left(\hat{\boldsymbol{n}}_{i}\right)=s_{\mathrm{CMB}}\left(\hat{\boldsymbol{n}}_{i}\right)+s_{\mathrm{kSZ}}\left(\hat{\boldsymbol{n}}_{i}\right)$.

We are focusing on the intrinsic contaminants whose subtraction cannot be improved by additional observations at different frequencies and/or better sensitivities (such as the point source emission or the instrumental noise). Further, the symbol $s_{\mathrm{kSZ}}\left(\hat{\boldsymbol{n}}_{i}\right)$ in the equation above refers to the $\mathrm{kSZ}$ residual which remains after estimating the kSZ (and the peculiar velocity field) from the knowledge of the density distribution in that region of the Universe. We shall see that the requirements on the amplitude of these $\mathrm{kSZ}$ residuals are not too stringent, but must be inferred by other observations different from those in the CMB range. We are interested in the signal arising in the cluster's area, and therefore the application of a high-pass filter may be useful to minimize the contamination of the large scale signal. This filtering should leave some residuals which are smaller for smaller clusters/groups and lie typically at the level of 10-30\% the kSZ amplitude (see Fig. 6 in Hernández-Monteagudo et al. 2006).

There will be other residuals due to the presence of radio/IR point sources, but, in any case, these residuals share the same statistical properties than the kSZ residuals, and they will be regarded as the same: our goal is to set upper limits for them which enable us to track the growth of the ISW at late epochs. That is, we propose comparing the low $l$ multipoles of the CMB at the

\footnotetext{
5 Pan-STARRS' URL site:

http://pan-starrs.ifa.hawaii.edu/public/

6 DES's URL site:

http://www . darkenergysurvey . org/

7 PAU-BAO's URL site:

http://www.ice.csic.es/research/PAU/
}

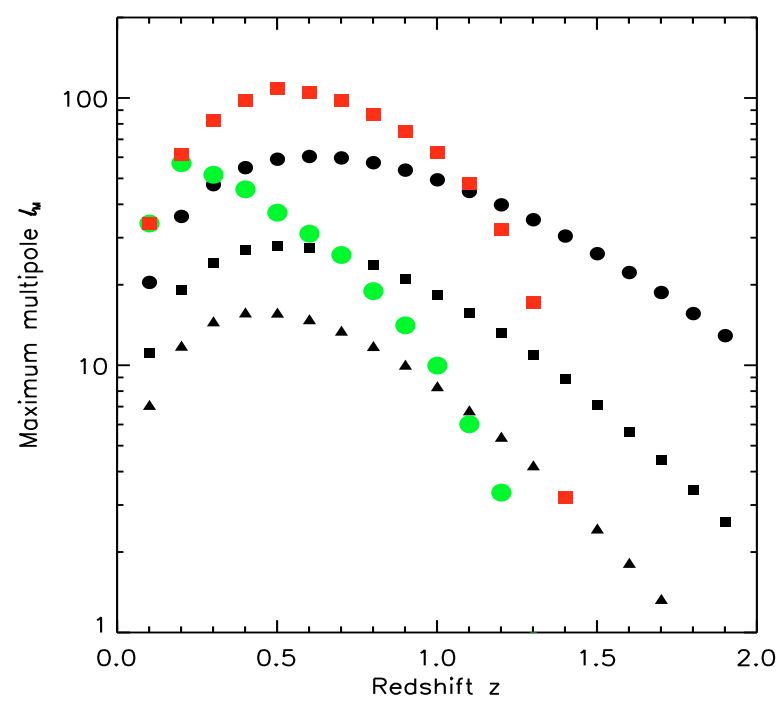

Fig. 6. Maximum multipole $l_{M}$ to which a discrete cluster sample in the full sky is sensitive (see Eq. (10)). The ISW is well contained within $l<30-40$.

high redshift cluster positions with the low $l \mathrm{CMB}$ multipoles measured from the whole sky. The difference must be due to the signal which arose between the clusters and the observer, i.e., the ISW.

If clusters are homogeneously distributed over the sky, it can be easily shown that the error in the estimation of a multipole $a_{l, m}$ in the set of pixels/clusters equals

$\Delta^{2}\left[a_{l, m}\right] \simeq \Omega_{\mathrm{cl}} \sigma_{\mathrm{kSZ}}^{2} \simeq \frac{4 \pi}{N_{\mathrm{cl}}} \sigma_{\mathrm{kSZ}}^{2}$

with $\sigma_{\mathrm{kSZ}}^{2}$ being the dispersion of the kSZ residual $s_{\mathrm{kSZ}}$ and $\Omega_{\mathrm{cl}}$ the solid angle assigned to each cluster. It is possible to make use of the $2 l+1$ modes in order to provide an estimate of the power at the multipole $l$. The error of such an estimate is given by

$\Delta\left[C_{l}\right] \simeq \frac{\Delta^{2}\left[a_{l, m}\right]}{2 l+1}=\frac{4 \pi}{N_{\mathrm{cl}}} \frac{\sigma_{\mathrm{kSZ}}^{2}}{2 l+1}$,

and hence the $S / N$ for the ISW detection at a given multipole reads

$\left(\frac{S}{N}\right)_{l}^{2}=\frac{C_{l}^{\mathrm{ISW}} N_{\mathrm{cl}}(2 l+1)}{4 \pi \sigma_{\mathrm{kSZ}}^{2}}$.

The total $S / N$ is obtained after adding this contribution from $l=2$ up to $l=l_{M}$ given in Eq. (10):

$\left(\frac{S}{N}\right)^{2}=\sum_{l=2}^{l=l_{M}}\left(\frac{S}{N}\right)_{l}^{2}$.

In Fig. 6 we display the maximum $l$ accessible by a cluster population driven from the Sheth-Tormen (Sheth \& Tormen 1999) mass function in a WMAPV universe with $\sigma_{8}=0.817$. The width of the redshift shell equals $\Delta z=0.1$. Black circles account for all clusters above $10^{14} h^{-1} M_{\odot}$, whereas black squares and black triangles correspond to $M_{\min }=2,3 \times 10^{14} \mathrm{~h}^{-1} M_{\odot}$. We also display the maximum multipoles to be reached with the cluster surveys obtained under the nominal sensitivity of the experiment eROSITA: big green circles correspond to a survey with a flux limit equal to $1.6 \times 10^{-13} \mathrm{erg} \mathrm{s}^{-1} \mathrm{~cm}^{-2}$ in the X-ray band $[0.5,5] \mathrm{KeV}$ (all sky survey), whereas the big red squares 

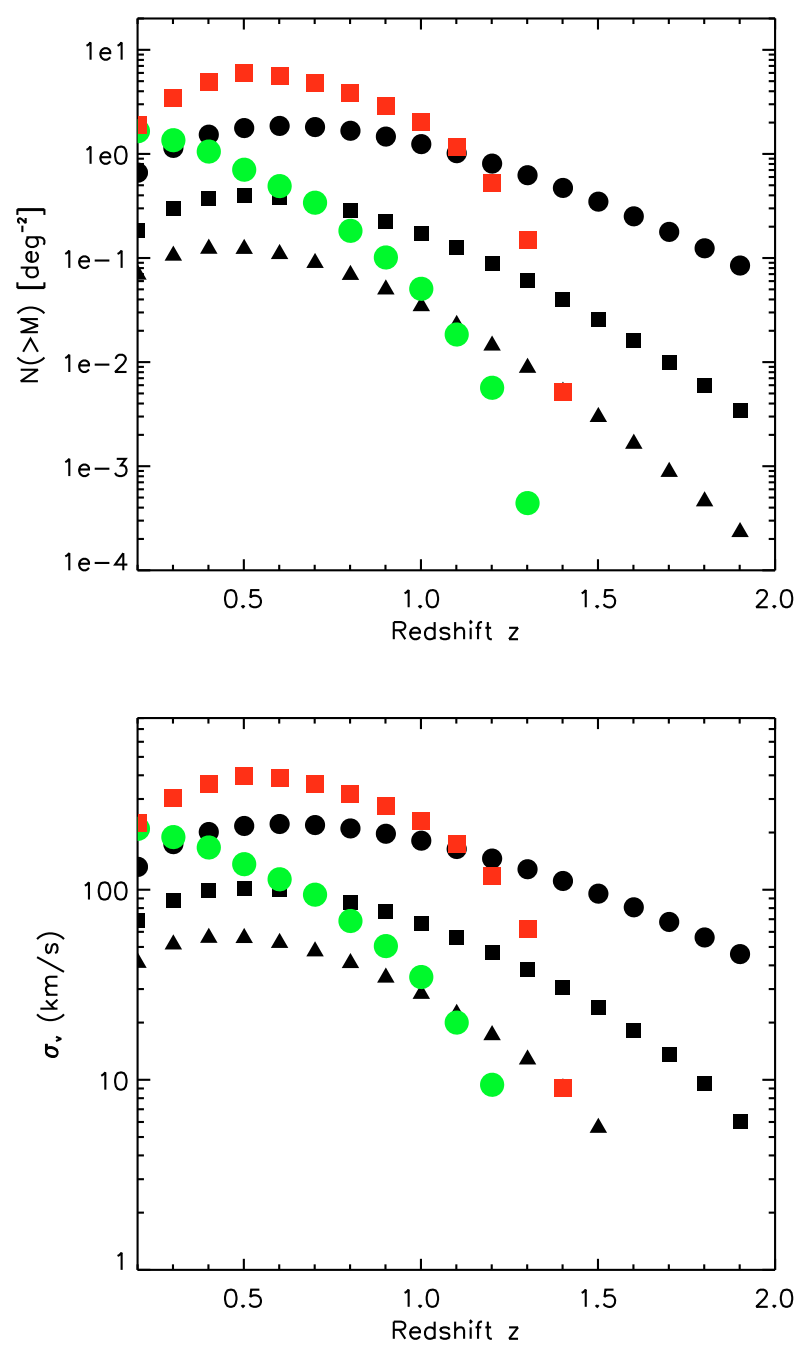

Fig. 7. Top: angular number density of different cluster populations in different redshift shells of the width $\Delta z=0.1$. Bottom: required errors in the $\mathrm{kSZ}$ recovery in order to obtain residuals three times below the ISW level $(S / N=3$ in Eq. (15)). In both panels, the symbol coding is identical to that in Fig. 6 as explained after Eq. (15).

correspond to a flux limit of $3.3 \times 10^{-14} \mathrm{erg} \mathrm{s}^{-1} \mathrm{~cm}^{-2}$ in the same range (wide survey, see http://www.mpe.mpg.de/erosita/ MDD-6.pdf for details). We remark that the ISW contains most of its power in the low multipole range, $l<20-30$.

In the top panel of Fig. 7 we display the actual angular density of those cluster populations at different redshifts, again for $\Delta z=0.1$. In the bottom panel, we display the required error in the peculiar velocity estimates $\left(\sigma_{v}=c \sigma_{\mathrm{kSz}}\right)$ in order to obtain a residual which is three times below the ISW level (i.e., $S / N=3$ ). The symbol coding is the same as in the previous plot. For the cluster sample of $M_{\min }=10^{14} h^{-1} M_{\odot}$ we require errors in the radial peculiar velocity of the order of $100-200 \mathrm{~km} \mathrm{~s}^{-1}$ in order to see the growth of the ISW at low redsfhits. These error requirements become more stringent when more massive cluster populations are used and when higher redshifts are to be probed. They are at the level of the actual linear prediction for the $\mathrm{kSZ}$ in clusters $\left(\sim 220 \mathrm{~km} \mathrm{~s}^{-1}\right)$, so a priori are not too stringent. However, we must remark that these are the upper limits for the total errors and should account for not only kSZ residuals, but also for all other types of possible contaminants and systematics. Note as well that the error on the kSZ recovery $\sigma_{v}$ is inversely proportional to the required $S / N$.

\section{Constraints on the cosmological dipole}

Let us assume now that we include the dipole in the analyses to be performed at the positions of the cluster distribution. The same requirements which allow the tracing of the ISW growth should permit us, a priori, to impose constraints on the intrinsic cosmological dipole of the same order, i.e., at the level of a few tens of $\mu \mathrm{K}$. Indeed, if we use Eq. (15) to impose $(S / N)_{l=1}=1$ with $\sigma_{\mathrm{kSZ}} \sim 100 \mathrm{~km} \mathrm{~s}^{-1}$ and $C_{l=1}^{\mathrm{ISW}} \sim 400(\mu \mathrm{K})^{2}$, we obtain $N_{\mathrm{cl}} \sim 4 \times 10^{3}$. This is a relatively modest number of clusters: by including all groups and clusters present in the different redshift shells, the constraints on the cosmological dipole would improve even further and could eventually yield a detection. With 4000 clusters alone, the constraint of $\sim 20 \mu \mathrm{K}$ is between one and two orders of magnitude below the upper limit of the cosmological dipole which can be inferred from our modelling of the motion of the local group.

A natural question that arises in this context is how the subtraction of the measured dipole (which includes both the intrinsic cosmological dipole and the one induced by our peculiar motion) affects our estimates of the intrinsic dipole in the positions of galaxy clusters. Let us assume that the total dipole measured on the whole sphere is

$a_{1, m}=a_{1, m}^{\mathrm{intr}}+a_{1, m}^{\mathrm{vobs}}$,

built upon components of both the intrinsic cosmological and the observer's velocity dipoles. If, for the time being, we neglect all $\mathrm{kSZ}$ residuals in our subset of pixels containing clusters, ideally the total dipole measured at cluster positions would be

$a_{1, m}^{\mathrm{cl}}=a_{1, m}^{\mathrm{intr}}\left(1-\left\langle\tau_{\mathrm{T}}\right\rangle\right)+a_{1, m}^{\mathrm{vobs}}$,

but in practice it will be

$a_{1, m}^{\mathrm{cl}, r} \simeq a_{1, m}^{\mathrm{intr}}\left(1-\left\langle\tau_{\mathrm{T}}\right\rangle\right)+a_{1, m}^{\mathrm{vobs}}+\sum_{l^{\prime}, m^{\prime}} \epsilon_{l^{\prime}, m^{\prime}} a_{l^{\prime}, m^{\prime}}$

where the last component accounts for the errors introduced by our discrete cluster (pixel) distribution, and $\left\langle\tau_{\mathrm{T}}\right\rangle$ is the average cluster optical depth. Since computing the dipole in our pixel subset is a linear operation on the map, the errors in the dipole estimation will also be linear in the different multipoles conforming the map to the whole sphere. This leakage of power from different multipoles to the estimated dipole moment $(1, m)$ is described by the geometrical vector $\epsilon_{l^{\prime}, m^{\prime}}$, which is only dependent on the angular distribution of the cluster set and can be computed with high precision. Note that we are ignoring the relativistic effects associated to the motion of the observer (Chluba et al. 2005). If, as is usually done in a CMB analysis, the total dipole measured in the entire sphere is subtracted, we would be left in the cluster pixel set with

$\delta a_{1, m}^{\mathrm{cl}} \simeq-\left\langle\tau_{\mathrm{T}}\right\rangle a_{1, m}^{\mathrm{intr}}+\sum_{l^{\prime}>1, m^{\prime}} \epsilon_{l^{\prime}, m^{\prime}} a_{l^{\prime}, m^{\prime}}$.

That is, the rms error on the limits for the intrinsic cosmological dipole will be of the order

$\left(\Delta\left[a_{1, m}^{\mathrm{intr}}\right]\right)^{1 / 2} \sim \frac{\sum_{l^{\prime}>1, m^{\prime}} \epsilon_{l^{\prime}, m^{\prime}} a_{l^{\prime}, m^{\prime}}}{\left\langle\tau_{\mathrm{T}}\right\rangle}$. 
According to this expression it becomes critical to choose a convenient cluster/pixel set that fulfills $\epsilon_{l^{\prime}>1, m^{\prime}} /\left\langle\tau_{\mathrm{T}}\right\rangle \ll 1$, so that interesting limits on $a_{1, m}^{\text {intr }}$ can be set. If the $\mathrm{kSZ}$ removal is accurate and/or the large angle power of the $\mathrm{kSZ}$ is negligible, the residuals of our ISW estimation will be dominated by those given in Fig. 6 of Hernández-Monteagudo et al. (2006). In that case the average residual dipole for a population of $\sim 500$ galaxy clusters should be of the order of $30 / \sqrt{N_{\mathrm{cl}}} \times \sqrt{3} \sim 2 \mu \mathrm{K}$ if we assume an angular size of $20-30$ arcmins, but lower $(\sim 0.4 \mu \mathrm{K})$ for smaller sized ( $\sim 3$ arcmin) clusters. These figures ignore the impact of instrumental noise. However, Kashlinsky et al. (2009) were able to achieve a sensitivity level of a few $\mu \mathrm{K}$ in each component of the dipole computed on a $\sim 600$ cluster set with WMAP data. A residual dipole amplitude of $\sim 2 \mu \mathrm{K}$ on a cluster set of $\left\langle\tau_{\mathrm{T}}\right\rangle \sim 5 \times 10^{-3}$ would already correspond to a constraint on the primordial dipole at the level of $\sim 450 \mu \mathrm{K}$, which is well below the observed dipole. For the same cluster set, a residual amplitude of $\sim 0.4 \mu \mathrm{K}$ (as quoted for $\sim 3$ arcmin clusters) should yield a constraint at $\sim 77 \mu \mathrm{K}$, which is close to the expected value of the intrinsic dipole $(\sim 30 \mu \mathrm{K})$. We conclude that ongoing CMB experiments like Planck, ACT or SPT should be able to set strong constraints on the intrinsic $\mathrm{CMB}$ dipole.

\section{Discussion and conclusions}

Current CMB high angular resolution surveys like ACT or SPT are scanning the sky at frequencies in which the identification of galaxy clusters and groups should be possible via the tSZ and the kSZ effects. Nominally, these experiments should be able to detect via the tSZ all clusters more massive than $2 \times$ $10^{14} h^{-1} M_{\odot}$ at large significance, but many smaller clusters and groups should remain close to the detection threshold. Once clusters have been identified by their $\mathrm{tSZ}$ distortion, attempts to detect the kSZ in a subset of those systems can be conducted. The kSZ is our only probe for peculiar velocities in the high redshift universe, and those can be used themselves as probes for Dark Energy and missing baryons (Hernández-Monteagudo et al. 2006; Hernández-Monteagudo \& Ho 2009). In this context, a precise characterization and correction of all possible contaminants is critical. The impact of the bSZ effect on the kSZ amounts to $\sim 20 \%$ for $10 \%$ of the clusters and groups, and becomes more important for those objects with small radial projection in their peculiar motions. Provided that the Thomson optical depth of the cluster is known, this effect should be accurately predicted from background CMB observations (as those available from e.g. WMAP or Planck). The measurement of this effect is itself a measurement of the gas content of the object under study.

This effect mirrors the CMB intensity at the epoch of scattering, and this is relevant for secondary anisotropies which arise at late times (like the ISW effect): a detection of the bSZ effect in objects placed at different redshift shells would provide the picture of the growth of the ISW at recent cosmological times. The measurement we are proposing here is statistical, and therefore it does not require a high $S / N$ in each cluster (just in the same way as in Hernández-Monteagudo \& Sunyaev 2008, for the kSZ E polarization mode cross correlation). Our arguments here are therefore similar to those given in that work: cluster and group positions can be inferred from observations in a wide range of frequencies (optical, IR, X-ray, millimeter), many of which are using those objects for studying the nature of Dark Energy. The critical issue is the nature and the amplitude of residuals in the $\mathrm{kSZ} / \mathrm{CMB}$ estimation at the cluster positions. To what extent do errors in the IR/radio point source subtraction and/or in the characterization of the local peculiar velocity field actually endanger the ISW measurements? This should critically depend on whether those errors are systematic or not. If those residuals can be regarded as independent from cluster to cluster, the viability of this project should hinge exclusively on the actual precision with which the point source and $\mathrm{kSZ}$ residuals can be removed from the clusters' area. In this regard, Diaferio et al. (2005) studied the systematics that might arise when measuring kSZ fluctuations at a cluster's position. Peculiar velocity reconstruction algorithms based upon the local density field should be sensitive to the dark matter peculiar velocity only. Diaferio et al. (2005) showed that, after averaging within the cluster's area, clusters/halos moving faster than $100 \mathrm{~km} \mathrm{~s}^{-1}$ show small differences in their dark matter - gas peculiar velocities (around 10\%). However, for those objects slower than $100 \mathrm{~km} \mathrm{~s}^{-1}$ this correction reached the level of $90 \%$ and could pose a problem for the approach suggested here. In that same work it is also shown that similar uncertainties associated with peculiar motions of gas clumps and clouds within the intra cluster medium may give rise to differences as large as $\sim 120 \mathrm{~km} \mathrm{~s}^{-1}$ between the halo's dark matter average peculiar velocity and the gas peculiar velocity in different parts of the cluster. This aspect, however, should be alleviated to great extent after integrating the intensity over the cluster's area. Since the ISW contains most of its power at low multipoles $\left(l_{M}<10-20\right)$, targets may be chosen at a convenient distance in order to minimize the required sky coverage. A possible strategy would then consist of uniformly distributing $N_{\text {patch }}$ patches in the sky (with $\left.N_{\text {patch }} \sim\left(4 / \pi l_{M}\right)^{2}\right)$, lying a distance $\theta \sim 4 / \sqrt{N_{\text {patch }}}$ away and scanning deeply through each of those patches until finding a set of sources of high $S / N$ at different redshifts. This would improve the efficiency of the survey (since a minimum amount of an area would be scanned) at the expense however of improving the flux/mass thresholds shown in Fig. 7. Hence one would encounter here a trade-off between flux sensitivity and sky coverage. Whatever approach is finally chosen, it should also provide strong constraints on the cosmological dipole, which would be independent from those imposed from the local dipole and the local velocity flows. Let us also remark that this term has also been shown elsewhere (Khatri \& Wandelt 2009) to be responsible for the generation of some level of non-Gaussianity due to the in-homogeneity of recombination.

In this work we propose using for the first time the so-called blurring term in Thomson scattering for cosmological purposes. The small fraction of scattered off CMB photons which are deviated when crossing a galaxy cluster/group should provide information about what the $\mathrm{CMB}$ anisotropy field was like at the time of the scattering. If those objects are far away enough, the CMB at that epoch should lack the ISW component that has arisen recently, and this difference could a priori be picked up after removing all other signals present in the cluster with enough accuracy. Assuming that tSZ residuals are negligible, we find that a typical error of $100-200 \mathrm{~km} \mathrm{~s}^{-1}$ in the cluster peculiar velocity reconstruction is required for all clusters more massive than $10^{14} h^{-1} M_{\odot}$ in order to trace the growth of the ISW at late times. These errors are comparable with the linear expectations for the peculiar motions of those objects, which involves that $(i)$ the blurring correction to the kSZ is in general of relevance for the estimation of the latter and (ii) no very precise corrections for the $\mathrm{kSZ}$ are required. Those amplitudes roughly correspond to an error of $900-1800 \tau_{\mathrm{T}} \mu \mathrm{K}$ per cluster. The same level of errors would provide stringent constraints of the intrinsic cosmological dipole. Current and future large scale structure surveys like eROSITA, Pan-STARRS, DES, PAU-BAO, ACT or SPT should soon provide enough group and cluster candidates 
C. Hernández-Monteagudo and R. A. Sunyaev: Galaxy clusters as mirrors of distant Universe

at the relevant redshift ranges. Therefore, the critical point is the feasibility of an accurate enough $\mathrm{kSZ} / \mathrm{tSZ} /$ point source subtraction in future high resolution $\mathrm{CMB}$ observations.

\section{References}

Abramo, L. R., \& Xavier, H. S. 2007, Phys. Rev. D, 75, 101302 Bardeen, J. M., Bond, J. R., Kaiser, N., \& Szalay, A. S. 1986, ApJ, 304, 15 Benitez, N., et al. 2008, ApJ, 691, 241

Challinor, A. D., Ford, M. T., \& Lasenby, A. N. 2000, MNRAS, 312, 159 Chluba, J., Hütsi, G., \& Sunyaev, R. A. 2005, A\&A, 434, 811

Cooray, A., \& Baumann, D. 2003, Phys. Rev. D, 67, 063505

Diaferio, A., Borgani, S., Moscardini, L., et al. 2005, MNRAS, 356, 1477

Ettori, S., Morandi, A., Tozzi, P., et al. 2009, A\&A, 501, 61

Giodini, S., Pierini, D., Finoguenov, A., et al. 2009, ApJ, 703, 982

Hernández-Monteagudo, C., \& Ho, S. 2009, MNRAS, 398, 790

Hernández-Monteagudo, C., \& Sunyaev, R. A. 2008, A\&A, 490, 25

Hernández-Monteagudo, C., Verde, L., Jimenez, R., \& Spergel, D. N. 2006, ApJ, 643,598

Inogamov, N. A., \& Sunyaev, R. A. 2003, Astron. Lett., 29, 791

Hincks, A. D., et al. 2009 [arXiv: 0907.0461]

Itoh, N., \& Nozawa, S. 2004, A\&A, 417, 827
Kamionkowski, M., \& Loeb, A. 1997, Phys. Rev. D, 56, 4511

Kashlinsky, A., Atrio-Barandela, F., Kocevski, D., \& Ebeling, H. 2009, ApJ, 691, 1479

Khatri, R., \& Wandelt, B. D. 2009, Phys. Rev. D, 79, 023501

Kitaura, F. S., \& Enßlin, T. A. 2008, MNRAS, 389, 497

Peel, A. C. 2006, MNRAS, 365, 1191

Rephaeli, Y. 1995, ApJ, 445, 33

Sachs, R. K., \& Wolfe, A. M. 1967, ApJ, 147, 73

Sazonov, S. Y., \& Sunyaev, R. A. 1999, MNRAS, 310, 765

Seljak, U., \& Zaldarriaga, M. 1996, ApJ, 469, 437

Seto, N., \& Sasaki, M. 2000, Phys. Rev. D, 62, 123004

Sheth, R. K., \& Tormen, G. 1999, MNRAS, 308, 119

Sheth, R. K., \& Diaferio, A. 2001, MNRAS, 322, 901

Sunyaev, R. A., Norman, M. L., \& Bryan, G. L. 2003, Astron. Lett., 29, 783

Staniszewski, Z., Ade, P. A. R., Aird, K. A., et al. 2009, ApJ, 701, 32

Sunyaev, R. A., \& Zeldovich, Y. B. 1970, Ap\&SS, 7, 3

Sunyaev, R. A., \& Zeldovich, Y. B. 1972, Comments Astrophys. Space Phys., 4, 173

Sunyaev, R. A., \& Zeldovich, Y. B. 1980, MNRAS, 190, 413

Sunyaev, R. A., \& Zeldovich, Y. B. 1981, Astrophys. Space Phys. Rev., 1, 1

Vikhlinin, A., Kravtsov, A., Forman, W., et al. 2006, ApJ, 640, 691

Yoshida, N., Sheth, R. K., \& Diaferio, A. 2001, MNRAS, 328, 669

Zeldovich, Y. B., \& Sunyaev, R. A. 1969, Ap\&SS, 4, 301

Zeldovich, Y. B., \& Syunyaev, R. A. 1980, SvA Lett., 6, 285 